\title{
Bericht über die 57. Jahresversammlung der Deutschen Ornithologischen Gesellschaft 1907.
}

Verhandelt Berlin, Freitag, den 4. Oktober Abends 8 Uhr im Architekten-Vereinshause Wilhelmstr. 92 I.

Anwesend von Mitgliedern: Fräulein Snethlage und die Herren: Kollibay, Schalow, Heck, Reichenow, Haase, v. Treskow, Neumann, Hartert, v. Quistorp, Jacobi, Helm, Deditius, v. Dallwitz, Ehmcke, Paeske, Schillings, Thienemann, Frh. v. Schweppenburg, König, v. Lucanus, Gottschlag, Bünger, Voigt, F. Henrici, Hantzsch, Braun, Kuschel, Neunzig, le Roi, Graf Zedlitz, Krause, Schiller, Koske, Schnoeckel, Frh. v. Berlepsch, Dietrich.

Als Gäste nahmen teil die Herren: Plate, K. Kothe, Hans Werther v. Quistorp, F. E. Stoll (Riga), Staudinger, A. Brauer, E. Vanhöffen, H. Hocke, Herm. Grote, Erich Hoffmann, P. Kothe, Miethke, P. Krüger, M. Götzsche (Nyköbing a. Falster), G. Waeles, R. Priemol, Dr. Berger, Dr. Thümmel (Braunschweig), E. Stresemann, Ernst Nolda.

Vorsitzender: Herr Heck, Schriftführer die Herren K. Kothe und Haase.

Herr Heck begrülste die Anwesenden, sprach seinen Dank aus für das zahlreiche Erscheinen und die Hoffnung, dafs die Verhandlungen der schönen Wissenschaft zum Vorteile gereichen möchten.

Redner teilte hierauf mit, dafs der Gesellschaft durch den Tod entrissen worden sind: der Präsident der Gesellschaft, Herr Prof. Dr. Rud. Blasius, das Ehrenmitglied, Herr Prof. Alfr. Newton in Cambridge und das langjährige Mitglied, Herr A. Grunack. Herr Heck hebt die Verdienste der Verstorbenen, insbesondere des so plötzlich geschiedenen Präsidenten um die Gesellschaft und die Wissenschaft hervor. Die Versammlung ehrt das Andenken der Verstorbenen durch Erheben von den Plätzen.

Herr Reichenow legt die Tagesordnung fest und übermittelt schriftliche bezw. telegraphische Grüfse der Herren v. Tschusi, Wilh. Blasius, Nehrkorn, Borggreve, Heine, Reiser, Graf Berlepsch, Heinroth u. Frau. Herr Reichenow schlägt vor, zu stellvertretenden V.orsitzenden der Versammlung die Herren Schalow und Kollibay, zu Schriftführern die Herren Haase und K. Kothe zu wählen. Die Versammlung gibt ihre Zustimmung.

Nach ferneren geschäftlichen Mitteilungen durch den Generalsekretär erteilt der Vorsitzende Herrn Prof. Koenig das Wort:

Herr A l. Koenig berichtet zunächst über eine Locustella luscinioïdes, die er aus der Rheinprovinz am 21. Juli 1904 erhielt und die im Vergleich mit ungarischen Stücken auf dem Rücken grauer ist. Dieser Vogel wurde in Krickenbeck, dem Besitztum des Grafen Schaesberg nahe der holländischen Grenze durch Hans Freiherr v. Geyr-Schweppenburg entdeckt, weshalb der Vortragende 
diese Art subspecifisch fafst und sie zu Ehren des Entdeckers trinär Locustella luscinioïdes Geyri benennt.

Sodann schildert Hr. Koenig eine Reise in die arktischen Gewässer im Sommer 1905, und eine wissenschaftliche Expedition nach der Bäreninsel und Spitzbergen im Sommer 1907, deren Schilderung hier im Wortlaut folgt:

\section{Hochverehrte Herren!}

Im Sommer des Jahres 1905 unternahm ich nach einer glücklich überstandenen Operation mit meiner Frau eine sogen. Vergnügungsreise in die arktischen Gewässer. Da ich nun einmal prinzipiell nie ohne Flinten, Scalpelle und Arsenikseife reise, brachte mir auch diese in der Tendenz nur auf die Touristen zugeschnittene Fahrt eine Summe grofser Forscherfreuden. Durfte ich doch die wunderbare arktische Vogelwelt zum ersten Male sehen und kennen lernen! Während ich auf derselben fleifsig den Vater Heuglin studierte, lugte ich allseitig scharf aus und erblickte Springwale, Delphine und Seehunde, aber auch - und das interessierte mich natürlich am meisten - eine ganz eigenartige Vogelwelt. Wie war ich entzückt, als ich die langen Reihen der Lummen sah, die wie Perlenschnüre hintereinander zogen, oder auf dem Wasser schwimmend dicht vor dem Buge unseres Dampferkolosses in die Tiefe tauchend verschwanden. Dem Kiele aber folgten die Dreizehenmöwen, jene schaumgeborenen Kinder des Meeres selbst, im unaufhörlichen Ab- und Zufliegen, Auf- und Niederwallen. Am meisten jedoch, glaube ich, wird jedes Auge gefesselt werden durch den wunderbaren Flug des Eissturmvogels. Ohne jegliche Scheu vor dem Menschen und ohne Furcht vor dem dahinschnaubenden Riesendampfer gleitet dieser Taucher der Lüfte dicht an den Planken des Schiffes vorbei oder überfliegt gar den Vorderbug, zum Greifen nahe. Über die Wellen aber streicht er halb eulen-, halb schnepfenartig dahin und zwar so nahe, dafs man vermeint, er müsse die Spitzen seiner Fittiche netzen und damit dem Meere verfallen. Aber weit gefehlt: der Vogel kennt genau den Abstand zwischen sich und dem Wasser und die Muskulatur seinerVorderextremitäten funktioniert so hervorragend, dafs man niemals auch nur ein Tippen der Schwingen auf der Wasserfläche wahrnehmen wird.

Aber das Sehen allein genügt dem Forscher bekanntlich nicht. Er mufs die Gegenstände seiner Bewunderung mit der Hand fühlen, fassen und ergreifen können! Geradezu entlastet kam ich mir daher vor, als der Dampfer im Bellsund von Spitzbergen nach 2 tägiger Fahrt im nördlichen Eismeer die Anker niederrasseln liefs und ich so die Śpannungen meiner Sinnesorgane auslösen konnte.

Ich stieg in den Nachen und im Umsehen war derselbe gefüllt von den begehrenswertesten Vögeln der hohen Arktis: Teisten und Lummen, den niedlichen Krabbentauchern, Eiderenten, aber auch Seeschwalben, Dreizehenmöwen und Eissturmvögeln. Wer 
nie die Freuden empfunden hat, welche einem Forscher das Schiefsen und Erlangen neuer, bisher für ihn noch ungekannter Arten bringen, der freilich kann den Inbegriff der Herzenswallung nicht verstehen, - derjenige aber, der selbst jene Freuden durchkostet hat, der wird es begreifen können, in welchem Stadium des Glückes und der Freude ich mich befand, als ich in meinem beutebeladenen Nachen zum Dampfer zurückkehrte. Nur knappe 6 Stunden waren für den Bellsund ausgeworfen, eine leider natürlich viel zu kurze Zeitspanne, um die Reichtümer dieses für die Vogelwelt so begünstigten Fjordes auch nur einigermafsen erschöpfend kennen zu lernen. Und doch war es mir beschieden, kaum in 10 Minuten nach dem Betreten einer kleinen Insel daselbst einen für den ganzen Spitzbergen-Archipel neuen Brutvogel festzustellen, nämlich die Trauerente (Oedemia nigra). Bei meiner Annäherung strich die Ente flatternd vom Neste, wurde dabei geschossen und der kostbare Fund eines frischen Geleges eingeheimst.

Neue und reiche Freuden brachte mir der 36-stündige Aufenthalt in der Adventbai, den ich zunächst dazu benutzte, eine ganze Nacht, die in der Lichtfülle zu jenen Zeiten bekanntlich nahezu gleichwertig ist mit dem Tage, in dem Kahne auf dem Wasser zuzubringen. Ich schofs Dreizehen- und Bürgermeistermöwen, Eissturmvögel, Papageitaucher, Teisten und Dickschnabellummen, auch eine Reihe von der langschwänzigen Raubmöwe, sowie als Glanzobjekt der ganzen Reise eine wunderschöne Elfenbeinmöwe. Eine kurze Ruhe an Bord, - dann weiter hinaus auf's Meer, aber auch an's Land und in die Berge. Viel zumuten durfte ich mir noch nicht - da die Schnittwunde noch nicht genügend vernarbt war - aber ich fand doch allerlei Schönes, so das allerdings stark bebrütete Gelege vom Seestrandläufer (Tringa maritima) u. A. Es war spät, als ich auf unser schwimmendes Hotel zurückkehrte. Die Musikkapelle, welche so lustig vorher gespielt, hatte ihre Weisen längst eingestellt, aber die vergnügte an 300 Personen starke Gesellschaft erging sich - es ist unglaublich zu sagen - die Herren im Smoking und weifsen Pantalons - die Damen in hocheleganter decolletierter Toilette auf Deck, um die Mitternachtssonne zu geniefsen, die in ihrer ganzen Majestät vom Himmel niederstrahlte, als ob sie für uns bestellt worden wäre.

So dankbar ich auch für die erlebten Jagd- und Forscherfreuden war, so wehmütig war es mir doch zu Mute, als ich die schrille Pfeife des Kapitäns vernahm, welche die Abfahrt des Schiffes von Spitzbergens Küste verkündete. Ein grofser Herzenswunsch griff in mir Platz: Spitzbergen wiederzusehen, wenn möglich aber unter anderen Verhältnissen, die mich mehr Herr meiner Zeit sein liefsen. Was ich damals gewünscht, aber kaum zu fassen gewagt habe, hat sich mir in diesem Jahre glänzend erfüllt. Ich konnte einen norwegischen Dampfer zu meiner eigenen Verfügung erhalten, welcher mit grofser Sachkenntnis von Herrn Ingenieur 
de Gisbert in Hamburg geschartert wurde. Dieser Dampfer „Erik Jarl" mit Namen, d. h. Herzog, Erich gehörte einer norwegischen Küstendampfergesellschaft an und war für den Personenverkehr bestimmt. Er hat sich auf der ganzen Reise trotz seiner geringen Gröfse (er hatte nur einige einhundertundsiebzig Tonnen Rauminhalt) geradezu glänzend bewährt, indem er nicht nur den oft sehr gefährlich werdenden Eisschollen wacker Stand hielt, sondern auch unter den schwierigsten Verhältnissen sich immer wieder von seinen Wunden erholte und den auf der Rückreise einsetzenden, starken Sturm siegreich überwand, sodafs er wegen seiner Leistungen sogar von vielen Riesendampfern wirklich beneidet wurde. Beneidet aber konnte er mit Recht werden; war er doch der einzige in diesem Jahr (aufser den Waldampfern), welcher die ganze Westküste Spitzbergens bis hinauf zur Moffeninsel besuchen und die zahlreichen Baien und Fjorde anlaufen konnte!

Die Vorbereitungen zur Reise waren rasch getroffen, die Kisten bald gepackt. Das aus einigen 40 Collis bestehende Gepäck ergab das respektable Gewicht von weit über 2000 Kilogramm. Ich brachte diese „Kleinigkeit" persönlich nach Hamburg und liefs sie von dort per Schiff weiter nach Drontheim verfrachten. Alles ging glatt von Statten und als ich mit meinen Herren in Drontheim anlangte, war das Gepäck zur Stelle.

Auf dem Schiffe sah ich mich umringt von sehr lieben und sympathischen Menschen, die rasch in die dargebotene Hand, die Reise mitzumachen, eingeschlagen hatten. Da war zunächst ein Regierungsrat aus Düsseldorf, ein alter lieber Jugendfreund, der bis ins Kleinste hinein bemüht war, allen unsern Wünschen Rechnung zu tragen und vor der Abreise meiner diesmal mich nicht begleitenden Frau versprechen mufste, sie zu ersetzen, so gut es eben ging. Dieses Versprechen hat er glänzend gehalten. Nahm er mir doch in geradezu vornehmer Weise die kleinen Alltagssorgen ab, sodafs dadurch auch nicht der geringste Hauch von Verdrufs und Unannehmlichkeit an mich herantrat. Da war ferner der Medicus, zwei Herren in deren Adern Künstlerblut rollte und deren Pinsel in meisterhafter Weise die glänzenden Bilder mit den magischen Beleuchtungseffekten festzuhalten suchten, da auch - last not least - 2 Jünger der Wissenschaft, welche die Begeisterung für das Hehre und Schöne geradeso im Herzen trugen wie ich, und deren Wünsche und Wollen sich mit der Energie und dem Können voll paarten. Ihnen habe ich in erster Linie den glänzenden Erfolg meiner Spitzbergenreise zu verdanken. Für die Bearbeitung der geschossenen Vögel standen 4 Präparatoren zur Verfügung, die un ermüdlich tätig waren, aufserdem war da ein Büchsenmacher zur Instandhaltung sowie zum Eingreifen von nötig werdenden Reparaturen an unseren Gewehren. Aufser dem Kapitän war Herr de Gisbert durch eine liebenswürdige Persönlichkeit vertreten; ferner hatten wir bis Tromsöe einen Küstenlotsen an Bord, der 
von dort ab wiederum durch einen sehr bewährten Eislotsen ersetzt wurde. Auf meinen ganz besonderen Wunsch waren noch 2 Fangleute engagiert worden, von denen der eine - ein Lappe - ein berufsmäfsiger Kletterer aus den Vogelbergen Norwegens stammte und seine Sache auf der ganzen Reise vorzüglich machte. -

Nach diesen persönlichen Angaben gestatte ich mir denn $\mathrm{zu}$ dem eigentlichen, wissenschaftlichen Teile meines Vortrages überzugehen.

Schon in Norwegen selbst setzten natürlich unsere ornithologischen Beobachtungen ein.

Bei einem Ausfluge in die herrliche Umgebung von Christiania beobachteten wir Chrysomitris spinus, meist singend und balzend auf den höchsten Fichtenspitzen, Muscicapa atricapilla, Parus borealis $\mathrm{u}$. A.

In Drontheim kamen wir insofern einen Schritt weiter, als wir bei einem Besuche der herrlichen Lerfos-Wasserfälle bereits wertvolle Dunenjunge von Parus borealis und Turdus pilaris einsammeln konnten. Es wurden 35 Vogelarten beobachtet und notiert, nämlich Chrysomitris spinus, eine Turdus merula, die offenbar dort nicht häufig zu sein scheint, ferner Fringilla montifringilla, ein $\sigma^{\top}$ mit einer Raupe im Schnabel, also wohl fütternd - eine Kolonie Cotile riparia und viele Cypselus apus. Muscicapa grisola und atricapilla häufig, auch Ruticilla phoenicurus, Cinclus melanogaster, Anthus arboreus, Hirundo rustica und urbica, Sylvia hortensis und cinerea. Numenius arcuatus.

Am 12. Juni abends bestiegen wir unseren „Erik Jarl“ und dampften unmittelbar darauf ab. Dem Kiel des Schiffes folgten Silber- und Sturmmöwen, Mantel- und Heringsmöwen. Als wir so bei wunderbar schönem ruhigem Wetter in dem reizvollen Drontheimer-Fjord dahinfuhren, konnten wir uns nicht trennen von dem Anblick der uns umgebenden landschaftlichen Scenerie, zumal die Mitternachtssonne es gut mit uns meinte und unbeschreiblich schöne Beleuchtungseffecte hervorrief. Mehr noch als diese fesselte uns jedoch der Anblick der nordischen Vogelwelt. Mit heller Freude begrüfsten wir die ersten Eiderenten, deren schmucke Erpel unser ganzes Entzücken wachriefen. Wir sahen ferner Colymbus septentrionalis, vereinzelt Cepphus grylle, Graugänse - (welcher Species angehörig, konnten wir bei der flüchtigen Sicht nicht feststellen), Haematopus und einen Haliaëtus albicilla, den ersten und letzten, welchen wir auf der ganzen Fahrt zu sehen Gelegenheit hatten. Delphine und Seehunde tauchten oft dicht vor unsrem Schiffe auf. Mergus serrator und Phalacrocorax carbo sahen wir häufig, tags darauf auch graculus auf den Felsenbolmen in Mengen sitzen; Lestris parasitica einzeln.

Am 14. Juni ergötzten wir uns an einer Schar von Sterna macrura und minuta, die stofstauchend einem bestimmten Wasser- 
striche folgten; wir begegneten den ersten Tordalken und Lummen und sahen neben einer grofsen Anzahl Staren auch 2 Kolkraben und einen Schoof Oedemia fusca.

Am 15. waren wir in Tromsöe, jener Stadt, welche bereits arktisches Gepräge in ihrem ganzen Wesen und Umfange zeigt. Eine Excursion auf die Insel und an den See von Tromsöe brachte eine kostbare Reihe von Nestern mit frischen Eiern von Turdus pilaris, ein Nest mit Gelege von T. iliacus mit brütendem Vogel, den Herr v. Geyr in gröfster Geschicklichkeit mit der Hand gefangen hatte.

Natürlich wurde auch das Museum einer eingehenden Besichtigung unterzogen, dessen Conservator Herr Schneider und sein Assistent Paul Bjerkan uns darin herumzuführen die Freundlichkeit hatten. Wie gebannt, blieben wir alle an einer prachtvollen Gruppe arktischer, sehr starker und hochläufiger Füchse aus Norwegen stehen. Im unteren Saale war auch eine gröfsere Glasvitrine für die arktischen Vögel aufgestellt. Natürlich interessierten wir uns für die eingehende Durchsicht der SpitzbergenOrnis am meisten. Da erblickten wir Lagopus hyperboreus, Sundev. in einem $\sigma^{x}$-lichen und Q-lichen Stücke und einen Strepsilas interpres im Hochzeitskleide, der - wenn ich nicht irre - von Kolthoff auf Spitzbergen erlegt wurde.

In dem oberen Stockwerk ist die nordische Vogelsammlung nicht nur sehr reichhaltig, sondern auch sehr sauber und übersichtlich angeordnet und aufgestellt. Prachtvolle Suiten von Colymbus arcticus und glacialis, alle nordischen Möwenarten bis auf Xema und Rhodostethia in den verschiedenen Stadien gut ausgestopft; auch die Tetraonengruppe ist reich vertreten und sehr hübsch aufgestellt. An den kleineren Vögeln ist kein Mangel - sie alle sind mit sehr viel Mühe und Sorgfalt präpariert. - Mein ganzes Entzücken bildete eine herrliche Reihe prachtvoller Somateria Stelleri-Erpel, die alle im Tromsöer Bezirk geschossen waren. Mehr noch als diese begeisterte mich ein erythristisches Gelege von Larus argentatus. Dieses reizte mich so sehr, dafs ich dem Herrn Schneider 200 Kronen dafür bot. Es zuckte eigentümlich um seine Mundwinkel, als ich ihm den anscheinend überhohen Preis dafür nannte, und schon wurde er schwankend, ob er mir die Eier nicht doch dafür abgeben sollte, als die zufällig dazwischengeworfene Mitteilung, dafs diese Eier häufiger beim Tromsöer Kaufmann Tomasen käuflich zu haben sind, mich von meinem Angebot Abstand nehmen liefs. Natürlich suchten wir gleich diesen Biedermann auf, und siehe da! ein prachtvolles erythristisches Gelege mit noch 2 anderen Eiern vom vorigen Jahre lagen verstaubt in einer alten Cigarrenkiste. Als ich nach dem Preis fragte, meinte der Kaufmann, ich müfste 4 Kronen für jedes Stück zahlen und da ein Ei beschädigt war, wollte er mir dasselbe nicht anrechnen. So machte es 16 Kronen aus. 
Mit wichtiger Miene knapste ich dem Braven noch 1 Krone ab und wickelte die kostbaren Eier unter leisem Gekicher meiner Herren vorsichtig in Watte.

Am 15. Juni mittags 2 Uhr verliefsen wir Tromsöe und steuerten direct nach Norden auf die Bäreninsel los. Nach zweistündiger Fahrt empfing uns die offene See, die so glatt und ruhig war, wie sie unser im Dienst ergrauter Eislotse bisher noch kaum gesehen hatte. Hatten wir doch in Tromsöe eine Temperatur von $26^{\circ}$ Réaumur, wie sie in diesem Monat geradezu unmöglich für dort erscheint. Auf dem Dampfer hatten wir am 16. Juni auf hoher See noch $14^{\circ}$ Celsius. In der Nacht wetterleuchtete es mehrfach und ein leiser Donner grollte in der Ferne. Es war unheimlich schwül für jene Breite! Im Tromsöer Fjord tummelten sich viele Alca torda, aber beim Verlassen der Küste verschwanden sie mehr und mehr und machten den Lummen Platz. Wir sahen die ersten Fulmarus und Larus glaucus, sonst Rissa, Mormon und Lestris parasitica.

„Die Bäreninsel ist verpackt in Eis und dichte Nebel umwallen das Eiland. Hinzukommen ist unmöglich." So etwa lauteten die Antworten, welche man uns in Tromsöe auf unsere Fragestellung der heurigen Eisverhältnisse gab. Aber wir liefsen uns dadurch nicht einschüchtern: wir sahen selbst zu und fanden $\mathrm{zu}$ unsrer grofsen Verwunderung, dafs grade das Gegenteil der Fall war. Als die Umrisse der Bäreninsel am Nachm. des 16. Juni sichtbar wurden, vermifsten wir - natürlich zu unsrer Freude - das Packeis, und von Nebel war erst recht nichts zu sehen. Wunderbar klar lag die Insel vor uns, umgeben von einigen Eisschollen, auf denen Bürgermeistermöven und viele Lummen safsen. Das sah reizend aus, wir waren wie elektrisiert davon und konnten uns nicht trennen von dem Anblick, den jede Minute in veränderter Form unseren entzückten Augen bot. Fieberhaft vibrierte uns das Blut in den Adern und nur die Tatsache, dafs Mitternacht längst vorüber war und der Morgen uns frisch und kräftig begrüfsen mufste, liefs uns die erregten Nerven beschwichtigen und Ruhe zum neuen Tageswerke finden.

Bewamst und hochbestiefelt standen wir in aller Frühe auf Deck. Rasch verteilten wir uns in 2 Ruderboote und eröffneten gleich nach unserer Abfahrt ein wahres Bombardement auf die uns entgegenkommenden Lummen und Möwen.

Über die Lage und die geographischen Verhältnisse der Bäreninsel will ich mich hier nicht weiter verbreiten, dagegen die dortige Vogelwelt namentlich vorführen und die darüber vorliegenden Arbeiten einer Kritik unterziehen.

Swenander, der wohl die beste Arbeit über die Ornis der Bäreninsel geliefert hat, zählt 16 Brutvögel auf, die ich als solche vollauf bestätigen kann. 


\section{Plectophanes nivalis, (L.).}

Der Schneeammer ist auf der Bäreninsel bei weitem nicht so häufig wie auf Spitzbergen. Wir schossen jedoch eine genügende Anzahl als Belegstücke; Nester und Gelege fanden wir auf der Bäreninsel nicht, sahen aber die $\sigma^{x} \sigma^{x}$ Mitte Juni in voller Balz. Zeitweise, wahrscheinlich im ersten Frühling und Herbst, müssen grofse Schwärme von Schneeammern auf der Bäreninsel verweilen, da wir ihren Kot massenhaft auf dem Klippenmoos fanden.

\section{Tringa striata, L. $1766=$ maritima, Gm. 1788 ex Brünn.}

Ist häufig auf der Bäreninsel. Es liegen Vögel und ein guterhaltenes Gelege vor. Der Mageninhalt wird noch genau untersucht werden. Soviel ist aber gewifs, dafs der Seestrandläufer vorwiegend animalische Kost $\mathrm{zu}$ sich nimmt. Die im Magen aufgefundenen Süfswasseralgen werden wohl nur zufällig dahineingelangt sein und wohl kaum seine eigentliche Nahrung ausmachen, wie Römer und Schaudinn glauben. Das ist schon von Swenander rectificiert worden.

\section{Sterna macrura, Naum.}

Überall, namentlich aber im Norden der Insel auf kleinen Inselchen in Süfswasserseen brütend. Um Mitte Juli hatten sie durchweg noch frische Eier. Wir sammelten eine ganze Menge, viele $\mathrm{zu}$ zweien, einige aber auch $\mathrm{zu}$ je einem Ei im Gelege drei Stück im Gelege haben wir niemals gefunden.

\section{Rissa tridactyla, (L.).}

Bei unserer Ankunft auf der Bäreninsel hatten die Dreizehenmöwen durchweg Eier, aber auch schon kleine Dunenjunge. Das Gelege besteht meistens aus 2 Stück, doch findet man mitunter auch 1 Ei. Die Eier sind sehr charakteristisch-stumpf und unterliegen weitgehenden Variationen. Der ohrenbetäuhende Lärm, den eine gröfsere Kolonie dieser Vögel hervorbringt, ist unbeschreiblich, die hochrandigen Nester stehen dicht beieinander und werden von den Besitzern auf's schärfste verteidigt. Mitte Juli fanden wir schon erwachsene Junge in den Nestern, die natürlich sorgfältig präpariert wurden. In den Kolonien sieht man nur alte, geschlechtsreife Individuen, auch auf dem Meere werden nur ganz selten einjährige Stücke wahrgenommen.

\section{Larus glaucus, Brünn.}

Wir haben die Bürgermeistermöwe auf der Bäreninsel als häufigen Brutvogel kennen gelernt. Sie legt ihre aus Moosstücken locker gefertigten, umfangreichen Nester meist auf der Höhe der Bergkanten, aber auch auf kleinen Inselchen an, - und durchaus nicht selten nur einige Fufs über dem Meeresspiegel auf nur wenig höher gelegenen Klippen und Erdböschungen. Swenander be- 
zeichnet diese Beobachtung, welche bereits 1864 von der schwedischen Expedition und sodann in der Fauna Arctica von Römer \& Schaudinn erwähnt wird, als nicht zutreffend. Jedoch befindet sich Swenander darin im Irrtum. Wir haben eine ganze Reihe von Nestern fast unmittelbar am Seestrande gefunden und daraus Eier und Dunenjunge genommen, die Zahl der Eier ist in der Regel 3, man findet jedoch auch sehr häufig 2. Ein einzioes Mal fanden wir 4 Eier im Gelege, was wohl als grofse Seltenheit zu bezeichnen ist, um so mehr, als alle 4 angebrütet waren. Die wunderhübsch getüpfelten Dunenjungen sammelten wir in allen Stadien ein; auch 1 jährige und 2 jährige Exemplare schossen wir vereinzelt, namentlich im Russenhafen, wo wir grofse Scharen von Bürgermeistermöwen an Walfischkadavern antrafen.

\section{Lestris parasitica, L. Schmarotzerraubmöwe.}

Ist sehr häufig auf der Bäreninsel. Im letzten Junidrittel scheint die Fortpflanzung einzusetzen. Bei unserem ersten Dortsein fanden wir noch keine Eier, bei unserer Rückkehr hoch bebrütete Eier und eben ausgefallene Dunenjunge. Wir erlegten auch die dunkele Form, die nur selten vorzukommen scheint, in mehreren Exemplaren.

\section{Fulmarus glacialis, L.}

Der Eissturmvogel brütet auf der ganzen Insel angesichts des Meeres auf Schutthalden und an schroffen Felshängen. Eine sehr grofse Kolonie befindet sich auf dem Gullfelsen, wo wir in kürzester Frist viele Dutzend Eier sammelten. Hellweifse und dunkelgraue Vögel brüten dicht neben einander. Ich möchte die hellen Stücke für ein höheres Altersstadium halten und die dunkelgrauen als junge Vögel ansprechen. Er legt natürlich stets nur $1 \mathrm{Ei}$, einmal fanden wir auch einen Vogel auf 2 Eiern brütend an, wovon jedoch $1 \mathrm{Ei}$ faul war und wahrscheinlich von einem anderen Vogel stammte, Vögel in den verschiedensten Färbungsstadien, Eier und Dunenjunge liegen in grofser Anzahl vor.

\section{Harelda glacialis, (L.).}

Vom Nordhafen ausgehend, fanden wir auf einem kleinen Inselchen eines gröfseren Süfswassersees mehrere Nester mit Eiern der Eisente und schossen auch die dazugehörigen Vögel. Leider aber haben wir kein vollständiges Gelege gefunden, obschon die Eier sehr spät (Mitte Juli) eingesammelt wurden. Viele Eisenten waren bereits in starker Mauser, sodals sie nicht fliegen konnten. Die $\sigma^{x} \sigma^{x}$ waren in ihrem eigenartigen dunkelschwarzbraunen Hochzeitskleide.

\section{Somateria mollissima, (L.).}

Im Norden der Insel auf Süfswasserseen Brutvogel - doch lange nicht so häufig wie in Spitzbergen. - Es liegen Vögel und Eier von dort vor. 


\section{Colymbus septentrionalis, (L.).}

Der rotkehlige Taucher darf als häufiger Brutvogel auf der Bäreninsel bezeichnet werden. Wir fanden ihn an den Sülswasserseen im Norden, wo wir eine genügende Anzahl Vögel und Eier einsammelten.

\section{Cepphus Mandti, Licht.}

Die Grylllumme oder Teiste, wie sie in Norwegen allgemein genannt wird, ist in dieser anscheinend gut ausgesprochenen Form auf der Bäreninsel nicht übermäfsig häufig. Sie brütet in Felsspalten, die meist hochgelegen ein Zukommen zu dem zweier Gelege sehr erschweren.

\section{Uria troile, (L.).}

13. Uria rhingvia, Brünn, Malmgr.

\section{Uria Brünnichii.}

Alle 3 Arten brüten auf der Bäreninsel. $U$. troile und rhingvia mehr in der Tiefe, Brünnichii auf der Höhe. Die Eier wurden aufs sorgfältigste artlich gesondert gesammelt. Da $U$. rhingvia niemals mit troile gepaart angetroffen wurde, halte ich erstere für eine gut abgeschlossene Subspecies von troile. Die Dunenjungen von Brünnichii unterscheiden sich gut von troile und rhingvia.

\section{Alca torda, L.}

Der Tordalk wird von Keilhau bereits für die Bäreninsel angegeben, obgleich dies auf einem Irrtum beruhen dürfte. Kolthoff ist der einzige, welcher 2 Exemplare dieses Vogels an der Bäreninsel erbeutet hat. Römer und Schaudinn berichten, dafs sie den Tordalken auf der Bäreninsel in nicht sehr grofser Anzahl beobachtet haben, „wo er mit den Lummen und Teisten zusammen in den Felsspalten des Vogelberges brüten soll." Auch schreiben sie ihm 2 Brutflecken ${ }^{1}$ ) zu, während Uria Brünnichii nur einen hat, wonach die Vögel im Dunkeln nach dem Gefühl bereits gut auseinander zuhalten seien. Dagegen geben Römer u. Schaudinn keinen Bericht über die daselbst erlegten Stücke. Und dies mufs uns mit Recht sehr stutzig machen. Wir sind der Meinung, dafs sich R. \& Sch. mit dem Tordalken auf der Bäreninsel einfach versehen haben. Es ist das so eine Sache mit der blofsen Angabe von ev. gesehenen Vogelarten. Sie berichten zwar, dafs sich der Tordalk von der Lumme durch einen höheren Schnabel mit weifsen Querbändern unterscheidet, und dafs er sich vom Fluge direkt kopfüber ins Wasser stürze, während die Lummen sich nur mit dem Bauch auf die Wasserfläche werfen und auf der-

1) In wie weit das richtig ist, kann ich vor der Hand nicht entscheiden, da mir Brutalken nicht zu Hănden gekommen sind. Es wäre aber sehr auffallend, da der Tordalk bekanntlich auch nur immer $1 \mathrm{Ei}$ im Gelege hat.

Der Vortragende. 
selben eine Strecke weit hinschnurren, - aber, aber - wer bürgt mir für die Richtigkeit dieser Beobachtung, zumal Römer \& Schaudinn keineswegs als Fachornithologen gelten können. Wir haben uns bei dem zweimaligen Besuche der Insel die erdenklichste Mühe gegeben, Tordalken aus den Lummenschwärmen herauszuerkennen, doch ist uns dieses nicht gelungen. Und unseren Scharfblicken wäre der Tordalk gewirs nicht entgangen. Wir müssen es daher vor der Hand aufs allerentschiedenste bezweifeln, dafs der Tordalk ein Brutvogel auf der Bäreninsel ist. Dafs sich zuweilen einige Stücke dahin verfliegen, ist gewifs nicht ausgeschlossen, der Beweis des Brütens aber auf der Bäreninsel mufs für diese Art erst erbracht werden.

\section{Mergulus alle, (L.).}

Der Krabbentaucher darf nicht als Massenbrutvogel für das Bäreneiland angegeben werden.

An den Klippen der Nordspitze brütet er vereinzelt - am 14. Juli wurde ein Ei eingesammelt, das noch nicht übermä fsig stark bebrütet war, bis jetzt $\mathrm{m}$. W. das einzige $\mathrm{Ei}$, welches von der Bäreninsel vorliegt.

\section{Mormon fraterculus, (L.).}

Erbeuteten wir in mehreren Exemplaren auf der westlichen und nordwestlichen Seite der Bäreninsel - wo wir auch aus den tiefen Spalten und Löchern deren Eier erhielten. Auf Spitzbergen kommt durchweg die grofsschnäbliche Form ( $M$. glacialis) vor, die indessen auch schon an der Bäreninsel beobachtet und geschossen worden ist.

Als zufällig nach der Bäreninsel verschlagen wurden bis jetzt folgende Vogelarten namhaft gemacht.

18. Loxia curvirostra, (L.)

von Malmgren beobachtet.

19. Strepsilas interpres, (L.).

Ein Exemplar vom Chef der Expedition des deutschen Seefischerei-Vereins unfern des Nordhafens erlegt.

20. Numenius phaeopus, (L.).

Ein verirrtes Individuum wurde tot auf der Bäreninsel von der deutschen Expedition gefunden (1898).

\section{Lestris pomatorhina, (L.).}

Bereits von Malmgren 1864 beobachtet, wurde von Swenander ein Stück am 7. Juli erlegt, unfern des Kap Elisabeth (altes $\sigma^{\top}$ ).

22. Anser spec.? worden.

Graugänse sind mehrfach auf der Bäreninsel beobachtet 


\section{Somateria spectabilis, (L.).}

Ebenfalls mehrfach beobachtet. erweitern.

Diese Liste kann ich teilweise bestätigen, teilweise sogar

Ich selbst erlegte eine Lestris pomatorhina auf der Nordspitze, ferner sahen wir eine Graugans, von der wir wohl vermuten möchten, dafs es Anser brachyrhynchus gewesen ist, die vielleicht durch erhaltenen Schaden veranlafst wurde, vom Weiterzuge nach Spitzbergen abzustehen. Von Somateria spectabilis schofs ich mehrere Stücke im Übergangskleide, auch beobachteten wir unverkennbar ein prächtiges adultes $\sigma^{x}$ im Fluge dicht an unserem Boote vorbeiziehend. Jedoch haben wir diese Art brütend nicht nachweisen können.

Erweitert wird die Anzahl der auf der Bäreninsel beobachteten Vögel durch folgende Arten:

\section{Aegialites hiaticula, (L.).}

Es wurden 3 Stück geschossen und zwar von Herrn Dr. le Roi, Baron v. Geyr und Regierungsrat Nolda. Die Testikel waren hochgeschwollen und der Eierstock gut entwickelt, sodafs die Annahme wohl berechtigt erscheint, dafs der Halsbandregenpfeifer daselbst gebrütet haben würde.

\section{Phalaropus fulicarius, (L.).}

Der breitschnäblige Wassertreter wurde von einem Mitgliede meiner Expedition (Herrn Joseph Waeles) in 2 Exempl. geschossen und zwar an einem Süfswassertümpel unweit des Nordhafens am 13. Juli. Am 14. schofs ich selbst 5 Stück dieser für die Bäreninsel ganz neuen Art an einem von dem gen. Herrn bezeichneten Süfswassersee, woran er tags zuvor die Vögel gesehen hatte. Obschon ich die Eier trotz eifrigster Suche nicht fand, bin ich doch der festen Ansicht, dafs diese Art für die Bäreninsel als Brutvogel $\mathrm{zu}$ bezeichnen ist.

\section{Oedemia nigra, (L.).}

An der Nordwestseite sahen und erkannten wir deutlich ein Pärchen dieser Art.

In der nächsten Umgebung des von dem deutschen Seefischereiverein errichteten Blockhauses im Nordhafen fanden wir am 13. und 14. Juli verschiedene Federn, die untrüglich als traurige Überreste folgender Arten deutlich $\mathrm{zu}$ erkennen waren. Die Träger derselben sind wohl von den im Blockhause sich eingerichtet habenden Füchsen gerissen worden. Es sind:

\section{Sturnus (vulgaris)?. \\ 28. Turdus merula, L. \\ 29. Turdus iliacus, L.}

Die zum Beweis mitgebrachten Federn können jederzeit angesehen und nachgeprüft werden. Um die Zahl 30 vollzumachen, 
kann ich berichten, daIs von einer nach uns fortfahrenden Jagdgesellschaft ${ }^{1}$ ) 3 Stück Anas crecca, L. geschossen wurden, von denen mir ein Balg zur Prüfung von einem Mitgliede der Reisegesellschaft freundlichst übersandt wurde.

\section{Sp it z bergen.}

Für den Spitzbergen-Archipel ist eine stattliche Reihe nordischer Vogelarten angegeben, so z. B. von Herman Schalow, der mit bewunderungswürdigem Fleifse und grofser Sachkenntnis die Literatur darüber zusammengestellt hat ${ }^{2}$ ), 50 Species. Indessen sind viele derselben noch recht zweifelhaft, so dafs noch deren Bestätigung erst abgewartet werden mufs.

Römer und Schaudinn führen in ihrer schönen Arbeit (Fauna Arctica, Band I, Jena, Verlag von Gustav Fischer 1900) 28 Arten auf, die auch wir bestätigen konnten.

Es sind dies:

\section{Plectrophunes nivalis, (L.).}

Überall häufig, nicht selten hoch auf die Berge hinaufgehend. Es wurde ein wertvolles Material von Brutvögeln, Nestern und Eiern zusammengebracht. In der letzten Junihälfte setzte das Brutgeschäft ein.

\section{Lagopus hyperboreus, Sundev.}

Die für Spitzbergen endemische und wie es scheint, gut modificierte Form des Schneehuhnes ist von uns mit grofsem Eifer gesucht worden. Obsch on wir auf den Inseln und auf Prinz Carl Vorland sehr häufig Spuren vom Schneehuhn fanden (vom Fuchs gerissene Winterstücke und die Losung), haben wir diese ausgezeichnete Art doch nur ganz vereinzelt zu Gesicht bekommen. Diejenigen Stücke, welche wir in der Adventbai sahen, haben wir auch - allerdings unter grofsen Anstrengungen - geschossen - es waren $3 \sigma^{x} \sigma^{x}$ und 1 of. Die $\sigma^{x} \sigma^{x}$ standen in voller Mauser, das sehr vergilbte und abgenutzte Gefieder gab dem sonst so schönen Vogel ein schäbiges Ansehen. In der Sassenbai wurden von 2 Mitgliedern unserer Gesellschaft ebenfalls einige Schneehühner gesehen und zwar gegen Abend, nach denen wir am nächsten Morgen vergeblich suchten. $\mathrm{Zu}$ gewissen Zeiten (im

1) Auf dem Dampfer L ofoten, eine speciell auf Eisbären gerichtete Jagdexpedition, ebenfalls von Herrn de Gisbert in Hamburg vorbereitet.

2) Die Vogel der Arktis von Herman Schalow in Berlin. Erschienen in „Fauna Arctica“. Eine Z usammenstellung der arktischen Tierformen mit besonderer Berücksichtigung des Spitzbergen-Gebietes auf Grund der Ergebnisse der Deutschen Expedition in das Nördliche Eismeer im Jahre 1898. Unter Mitwirkung zahlreicher Fachgenossen, herausgegeben von Dr. Fritz Romer in Frankfurt a./M. und Dr. Fritz Schaudinn in Berlin, Band IV, Lieferung 1. Verlag von Gustav Fischer, Jena 1904. 
ersten Frühjahr und im Herbst) sollen die Schneehühner oft in grofsen Scharen die unteren Gebirgsstöcke besuchen, müssen sich aber zur Brutzeit aufserordentlich verteilen und zwar weitschichtig im ganzen Revier, so dafs das Auffinden derselben mehr dem Zufall anheimgegeben ist. Eine zerbrochene Eischale fand ich an der Gebirgsbasis von Prinz Carl Vorland an der Südspitze. Die Eier scheinen bis jetzt noch unbekannt zu sein. Obschon eine alpine mutus-Form, zeichnet sich das Schneehuhn Spitzbergens durch Gröfse und Stärke sehr aus.

3. Tringa striata, L. $1766=$ maritima, Gm. 1788 et Brünn.

Sehr häufig. Es wurden mehrere Gelege gesammelt.

\section{Phalaropus fulicarius, L.}

Verbreitet, jedoch nicht überall. Die Eier fanden wir, obschon wir uns mit grofser Mühe der Suche widmeten, nicht. Vögel schossen wir in genügender Anzahl.

\section{Numenius phaeopus, L.}

Von uns nicht beobachtet. Aufser dem einen von Römer und Schaudinn auf der Bäreninsel tot gefundenen Stücke, wird ein Exemplar von Cock erwähnt, das auf der Axel Insel in der Van Mijenbai in West-Spitzbergen ebenfalls verendet, aufgefunden wurde.

\section{Aegialites hiaticula, L.}

Ist neuerdings mehrfach beobachtet - auch von und in der Sassenbai gesehen und geschossen worden.

7. Sterna macrura, Naum.

Überall an der Seeküste und auf Inseln brütend.

8. Xema Sabinei, (Sab.).

Von dieser seltenen, hocharktischen Möwe sind 3 Stück von Römer und Schaudinn auf der Great Insel im N.-O. von Spitzbergen erbeutet worden, bis dahin die ersten vom Spitzbergengebiete sicher nachgewieseuen Exemplare. Wir fanden sie auf einer ganz von Treibeis umgebenen Insel in der Kingabai zum ersten Mal als Brutvogel in Spitzbergen. Herr v. Geyr entdeckte das Pärchen in hoher Luft, von wo ich das ơ durch eine verwundete Seeschwalbe herablockte und es dabei scho[s. Am nächsten Tage (7. VII. 07) erlegten wir auf derselben Stelle das 9 . Gleichzeitig fand Herr Dr. le Roi die kostbaren Eier, 2 an der Zahl. Das dritte Ei hatte das \& legereif im Schlauch.

\section{Pagophila eburnea, Gm.}

Die Elfenbeinmöwe wurde von uns nur ganz vereinzelt gesehen und zwar meistens im Treibeis. Sie scheint vorwiegend dem Osten und hohen Norden anzugehören. 


\section{Rissa tridactyla, (Linné).}

Häufiger Brutvogel im Spitzbergengebiet.

\section{Larus glaucus, Brünn.}

Die Bürgermeistermöve ist in Spitzbergen zwar nicht selten, aber bei weitem nicht so häufig wie auf der Bäreninsel. Brütet auf Holmen und auf den Höhen der Berge, welche steil zum Meere abfallen. Während wir die staffelförmigen Terrassen der Berge nach Gänsenestern absuchten, wurden wir häufig von dieser Möwe attackiert. Ein ganz weifs gefärbtes Stück schofs Herr v. Geyr auf einer kleinen Insel an Prinz Carl Vorland (Südspitze). Das seltene Exemplar stellt eine im höchsten Alter prononcierte Phase dar und ist keineswegs als ein Leucismus aufzufassen, da Füfse (Ruder) und Schnabel viel intensiver gefärbt waren, als bei den typischen Stücken. Bis jetzt sind m. W. nur 2 derartige Stücke bekannt, die beide im finnischen Museum von Helsingfors aufbewahrt werden und über die Palmén berichtet hat (v. Naumann, II. Auflage, XI. Band, pag. 270).

\section{Lestris parasitica, (L.).}

Die Schmarotzer-Raubmöwe kommt in der hellbäuchigen Form auf dem ganzen Spitzbergen-Archipel sowohl auf dem Festlande, als auch ganz besonders auf den Inseln häufig vor. Zweimal sahen wir auch die dunkele Phase. Ein dahingehöriger Vogel wurde von mir auf einer Insel im Eisfjord erlegt. Diese Art ist ein überaus gefährlicher Eierräuber auf den Holmen. Leider wurde nur ein frisches Gelege eingesammelt, dagegen eine reichliche Anzahl Vögel geschossen.

\section{Lestris catarrhactes, (L.).}

Die Riesenraubmöwe wurde von uns zweimal gesehen und deutlich erkannt (Van Keulenbai und Kingsbai).

\section{Lestris pomatorhina, Temm.}

Die Spatelraubmöwe wurde von uns mit Sicherheit nicht beobachtet.

\section{Lestris longicaudus, (Vieill.) 1819.}

In der Adventbai häufig, wo mehrere Stücke geschossen wurden; 1 Stück in der Sassenbai, sonst nur während der Fahrt an Spitzbergens Küste beobachtet.

\section{Alca torda, L.}

Der Tordalk ist bisher m.W. vom Spitzbergengebiete nicht nachgewiesen worden und mufs daher aus der Liste gestrichen werden. 


\section{Uria Brünnichii, (Malmgr.).}

Die Dickschnabellumme ist die einzige ihres Geschlechtes, welche auf Spitzbergen brütet. $U$. troile und $U$. rhingvia sind bis jetzt von dort nicht bekannt geworden.

\section{Cepphus Mandti, (Licht.)}

Die Eisteiste (C. grylle, L.) kommt in dieser anscheinend gut modificierten Species überall im Spitzbergengebiete als Brut-

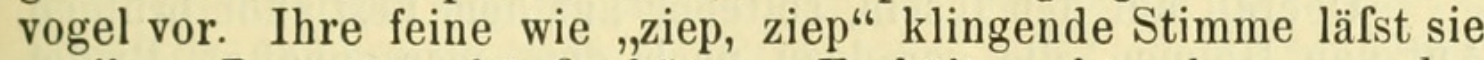
an ihrer Brutstätte häufig hören. Es hält recht schwer, zu den Eiern zu gelangen, die in Felsspalten tief verborgen sind.

\section{Mergulus alle, (L.)}

Der reizende Krabbentaucher brütet überall im W. Spitzbergens unter Felsblöcken und Steingeröll. Viele Eier eingesammelt zumal in der Magdalenenbai, wo unzählige Vögel brüten. Die Eier sind lichtmeergrün und zeigen nicht selten am stumpfen Pole braunrote Fleckenzeichnung. Dunenjunge erhielten wir nicht.

20. Mormon glacialis, Leach.

Nur die grofsschnäblige Form (glacialis) kommt in Spitzbergen brütend vor. Leider mifsglückten die Versuche, zu den Eiern zu gelangen.

\section{Colymbus septentrionalis, L.}

Ist an allen gröfseren Sülswasserseen Spitzbergens Brutvogel.

22. Fulmarus glacialis, L.

Der Eissturmvogel wurde von uns brütend auf Prinz Carl Vorland beobachtet. Über dem Wasser ist er gemein. Ein blendend weifses Stück - wahrscheinlich ein Albino - strich dicht an unserem Schiffe in der Sassenbai vorbei und konnte zu meinem gröfsten Bedauern nicht erlegt werden.

\section{Anser brachyrhynchus, Baill.}

Diese eigenartige Graugans wurde von uns überall im W. Spitzbergens auf den staffelförmigen Absätzen der Berge als Brutvogel angetroffen, besonders häufig war sie in der Van Keulenbai, auf Prinz Carl Vorland und auf den Inseln in der Kingsbai. Wir sammelten 93 Eier und schossen viele Vögel.

\section{Branta bernicla, L.}

Die Ringelgans ist im Gegensatz zur Graugans Tieflandbrüter. Wir fanden nur 3 volle Gelege auf der Forelands-Oie, die übrigen waren bereits von den rohen Robbenschlägern genommen worden, sodafs die der Eier beraubten Paare in Scharen auf den beiden Inseln safsen. Dunenjunge und alte Vögel wurden eingesammelt. 


\section{Branta leucopsis, (Bechst.)}

Die schöne Nonnen- oder Weifswangengans wurde von uns in der Adventbai als Brutvogel konstatiert, wo sie in den unzugänglichsten Felspartieen nistet. Wir hatten das Glück, 2 volle Gelege, welche hochbebrütet waren, am 30. Juni zu sammeln und 5 Vögel zu schiefsen.

\section{Somateria mollissima, L.}

Überall auf den Inseln, seltener auf dem Festlande brütend. Auf den Holmen, die geradezu nach dem massenhaften Brüten dieser Vögel Eiderholme benannt werden, wird den schönen Enten sehr bös nachgestellt. Es ist wahrhaft grauenerregend, wenn man an eine solche Wahlstatt kommt, wo Robbenschläger oder Walfänger gehaust haben. Ganze Strecken sind dort mit den nutzlos ausgehobenen Eiern und mit den herrlichen Vögeln bedeckt, die der grenzenlosen Verrohung dieser Leute anheimfallen mufsten.

\section{Somateria spectabilis, (L.).}

Die Prachteiderente wurde hier und da von uns gesehen und erlegt. Als Brutvogel trafen wir sie nur in der Adventbai, wo wir so glücklich waren, 2 Nester mit je 3 Eiern zu finden. Ebendort machte ich eine Dublette auf 2 prächtige Männchen.

\section{Harelda glacialis, L.}

Vereinzelt und nicht häufig beobachtet.

$\mathrm{Zu}$ dieser Liste käme dann noch die von mir 1905 aufgefundene Oeidemia nigra als Brutvogel hinzu, ferner

Phalaropus lobatus. (L.) den wir in einem Pärchen im Delta des Adventflusses erbeuteten und den ich dort als Brutvogel ansprechen möchte, - sowie

Mareca penelope, (L.) welche wir in einem prächtigen $\sigma^{x}$ als Einzelente unter einem grofsen Schwarm von gewöhnlichen Eiderenten auf einer der Goose-Inseln in der Sassenbai antrafen, leider aber nicht schossen, und endlich

Scolopax rusticula, L. wie die Federreste bezeugen, die ich vor einem Fuchsbau in der Sassenbai fand. Dieser Fund dürfte einer der interessantesten sein, da Scolopax circumpolar m.W. überhaupt noch nicht nachgewiesen worden ist.

Nach diesen Mitteilungen durfte ich mit den Ergebnissen meiner sechswöchentlichen Spitzbergenfahrt sehr zufrieden sein. Gar zu leid hatte es uns getan, dafs wir der diesjährigen abnorm schweren Eisverhältnisse wegen nicht nach dem Osten hatten gelangen können. Wie beneideten wir die Helgoland, der es im Jahre 1898 vergönnt gewesen war, nach König Karlland vorzudringen, ja sogar nach der Great Insel, die uns immer als ganz besonders besuchenswert vorgeschwebt hatte. Je länger je mehr sahen wir ein, von wie grofsem Glück diese Expedition begünstigt 
gewesen war, und fast wurde dieses Glück bei uns zum Tagesgespräch angesichts unserer allmählich zu Grabe getragenen Wünsche und Hoffnungen. Was hätten wir sonst da nicht noch Alles gefunden und gesammelt!!

Die Versammlung folgte den Ausführungen des Vortragenden mit lebhaftem Interesse und spendete reichen Beifall.

Am Schlusse zeigte Redner eine grolse Reihe prächtiger photographischer Aufnahmen von Landschafts- und Vogelbildern u. a aus dem von ihm besuchten Gebiete, welche die Versammlung ebenfalls aufserordentlich fesselten, und wofür sie durch Beifall dankte.

Herr Heck sprach dem Redner den herzlichen Dank der Versammlung aus und betonte, dafs sein Vortrag so verlockend gewirkt habe, dafs man gleich nach dem Norden reisen könnte.

Hierauf erhielt Herr Stoll das Wort zu einem Vortrage über einen Ausflug in Kurland. Der Vortragende besuchte am und im Rigaer Busen verschiedene Plätze und schildert das Vogelleben daselbst. Trotz der Greuel der Verwüstung in seiner baltischen Heimat sind dem Redner doch die weitgehensten $\mathrm{Zu}$ geständnisse seitens der Regierung gemacht worden, so dals ihm beim Sammeln keinerlei Hindernisse bereitet wurden. Auch dieser Vortrag war von photographischen Aufnahmen begleitet, die Landschaftsbilder, Vogelnester $u$. a. zeigten.

Wegen der vorgerückten Zeit wurde von einer Diskussion Abstand genommen.

Herr Heck schlofs die Versammlung mit einem Dank an Herrn Stoll für seinen interessanten Vortrag.

\section{Sonnabend, 5. Oktober.}

Um $9^{1} / 2$ Uhr versammelten sich die Teilnehmer im Zoologischen Garten, um unter Leitung des Direktors Prof. Heck den ungemein reichen Tierbestand und die geschmackvollen Anlagen und Bauten zu besichtigen. Insbesondere fesselten während des vierstündigen Rundganges die reich besetzten Vogelhäuser und Gehege, in denen viele auch in Museen noch seltene Spezies vorgeführt wurden und $\mathrm{zu}$ lebhaften Erörterungen Veranlafsung gaben. Bei Besichtigung des Eulen-Hauses nahm Hr. Reichen ow Gelegenheit, an einem von Herrn Prof. Vosseler aus Amani in Usambara (Deutsch-Ostafrika) dem Garten geschickten Uhu die Taufe zu vollziehen. Er nannte die neue Art Bubo vosseleri und gab folgende Diagnose:

Dem Bubo poensis sehr ähnlich, aber durch ockergelbbraunes Gesicht und grofse schwarzbraune Flecke auf den Seiten der Vorderbrust unterschieden; das aus weifsen Flecken gebildete Schulterband ist sehr deutlich.

Nach dem Run dgange vereinigte die Mitglieder ein gemeinsames Mittagessen im Restaurant des zoologischen Gartens.

Abends fand eine zwanglose Zusammenkunft im Restaurant Rheingold statt. 


\section{Sonntag, 6. Oktober.}

Verhandelt Berlin im Zoologischen Museum, Invalidenstr. 43. Anfang $9^{1} / 2$ Uhr Vorm.

Herr Kollibay eröffnet die Sitzung und erteilt Herrn Reichenow das Wort, der zunächst die Wahl des Vorstandes beantragt und Herrn Schalow als 1. Vorsitzenden, Herrn Kollibay als 2. Vorsitzenden in Vorschlag bringt. Herr Jacobi befürwortet den Vorschlag und beantragt Wahl durch Zuruf, welchem Antrage Folge gegeben wird. Herr Kolli bay erklärt sich mit Dank bereit, die Wahl anzunehmen, Herr Schalow ist noch nicht anwesend. Auch die Neuwahl des stellvertretenden Sekretärs ist notwendig, da Herr Matschie wegen anderer Verpflichtungen verhindert ist, das Amt weiter zu führen. Die Wahl fällt auf Herrn Heinroth, der schon längere Zeit das Amt vertretungsweise verwaltet hat. Aus dem Ausschufs scheiden die Herren Kollibay und Schalow, an ihre Stelle werden die Herren Heck und Parrot (München) gewählt. Herr Reichenow dankt den ausscheidenden Vorstandsmitgliedern, den Herren Heck und Matschie für ihre Mühewaltung.

Sodann wird die Wahl des nächsten Versammlungsortes beraten. Herr Reichenow schlägt vor, im nächsten Jahr in Rossitten zu tagen. Herr Thienemann äufsert sich freudig über diese Wahl und möchte auch Königsberg mit einschliefsen. Herr Braun bringt Danzig in Vorschlag, während Herr Henrici für Königsberg stimmt. Herr Reichenow hält es für zweckmälsig, beide Vorschläge zu vereinigen und die Versammlung zum einen Teil in Danzig, zum andern in Königsberg zu halten mit einem Ausfluge nach der Vogelwarte Rossitten. Der Vorschlag wird angenommen und dem Vorstand das Weitere anheimgestellt.

Herr Reichenow weist auf das von ihm gestiftete Ornithologen-Album hin und bittet die Ornithologen, die darin noch nicht vertreten sind, um ihr Bildnis.

Hierauf hält Herr Thien e mann einen längeren Vortrag über Ergebnisse der Vogelwarte Rossitten. An der Hand einer Karte von Europa, auf welcher die einzelnen Fundstellen gezeichneter Vögel eingetragen sind, weist er nach, dafs die Krähe (Corvus cornix), von welcher Art die meisten Stücke bis jetzt mit Ringen versehen worden sind, ihren Zug in breiter Front aus den russischen Ostseeprovinzen durch Norddeutschland bis ins nördliche Frankreich vollführt, von welchem die westlichste Krähe bis jetzt bekannt geworden ist. Wahrscheinlich brüten diese Krähen in den russischen Ostseeprovinzen. Nach den Beobachtungen des Redners an gezeichneten Stücken ziehen die Krähen denselben Weg zurück.

Der Vortragende geht sodann zur Besprechung der Erfolge mit gezeichneten Lachmöwen über, die recht interessant sind. So sind z. B. zwei fast gleichzeitig aufgelassene mit Ringen versehene Lachmöwen fast zur gleichen Stunde bei Wien erbeutet 
worden. Die äufsersten Fundstellen von Lachmöwen sind bis jetzt die Rhonemündung, die Pomündung (5 Stück), Spalato (Dalmatien). Sämtliche gezeichnete Lachmöwen sind bei Rossitten geboren, denn es wurden nur junge Exemplare zu diesem Zweck eingefangen. Der Redner erwähnt dann noch andere Arten, die mit Ringen versehen worden sind und die auch interessante Resultate gezeitigt haben, und geht hierauf zur Besprechung eines neueren Versuches über, zur Zeichnung von Störchen. Der Vortragende hat selbst eine grofse Zahl junger Störche im Neste mit Ringen versehen. Er schildert in humorvoller Weise die verschiedenen Erlebnisse, die er dabei gehabt, welche die Heiterkeit der Versammlung hervorriefen, hebt andererseits aber auch hervor, dafs er überall das bereitwilligste Entgegenkommen gefunden habe. Aber nicht nur selbst hat Redner Störche gezeichnet, sondern er hat auch andere in Ostpreufsen veranlafst, dies zu tun, und so sind über 1100 Storchringe bis jetzt ausgegeben worden. Die von Herrn Reichenow ausgesprochene Vermutung, dafs die Störche des östlichen und mittleren Europas im Herbst in südöstlicher Richtung ziehen, um die Winterherberge in Ostafrika aufzusuchen (s. Journ. Orn. 1907 S. 169), ist durch einen neuen Fall bestätigt. Der Vortragende legt eine Anzahl Läufe mit Ringen vor, darunter auch solche, die recht lange Zeit von den betr. Individuen getragen worden sind und keinerlei Beschädigungen aufweisen. Eine Ausnahme machte allerdings ein Ring, den Larus fuscus getragen hat, der sehr abgeschliffen war, nach Ansicht des Redners eine Wirkung des Sandes.

Herr Kollibay dankt Herrn Thienemann für seine interessanten Ausführungen und gibt dem Wunsche Ausdruck, die Vogelwarte möge materiell so gestellt werden, dafs sie ihren vielseitigen Aufgaben voll gerecht zu werden vermag.

Herr Schalow, der inzwischen erschienen ist, spricht seinen Dank für die Wahl aus, welche er annimmt und im Sinne seiner Vorgänger zu führen gelobt.

Herr Kollibay eröffnet die Diskussion über den Vortrag des Herrn Thienemann. Herr Heck vermilst süddeutsche Fundstellen von Corvus cornix, kein einziges Datum sei aus dieser Gegend, obgleich die Krähe dort sehr häufig ist. Er empfiehlt sich, mit süddeutschen Ornithologen in Verbindung zu setzen. Herr Kollibay erinnert daran, dafs die Krähe kein ausgesprochener Zugvogel ist und hält es für wahrscheinlich, dafs man es vielfach mit ansässigen Vögeln $\mathrm{zu}$ tun haben werde, die nur auf einer Vergnügungstur, nicht aber auf dem Zuge sich befinden. Herr Hartert spricht seine lebhafte Anerkennung über die Wirksamkeiten der Vogelwarte aus. Redner empfiehlt, auch andere Zugvögel aufser den Störchen zu zeichnen. Herr v. Lucanus hält den Prozentsatz der zurückgelieferten Krähen-Ringe für sehr hoch und spricht die Befürchtung aus, dafs der Ring hinderlich sein könnte und die gezeichneten Stücke so leichter eine Beute des Schützen werden. 
An Zimmervögeln hat allerdings Redner die Beobachtung gemacht, dafs Ringe garnicht beachtet werden. Herr Hartert meint, der Ring sei dem Vogel nicht hinderlich, der Zug gehe mit elementarer Gewalt weiter. Auch Herr Heck bestätigt, dafs Vögel des Zoologischen Gartens, die versuchsweise mit einem Ring versehen wurden, gar keine Notiz davon nahmen. Freiherr v. Berlepsch erwähnt, dafs am Neste gefangene und gezeichnete Vögel so verwirrt waren, dafs sie nicht wiederkamen und die Jungen verhungern liefsen. Redner empfiehlt die engen Ringe, damit sie die Vögel beim Brüten nicht stören. Herr Thienemann erwidert, dafs man es in Rossitten ohne Zweifel mit ziehenden Krähen zu tun habe, nicht mit ziellos herumstreichenden, wie er an der Art des Fluges u. a. glaubt bestimmen zu können. Den Versuch mit kleineren Vögeln hält Redner für wenig aussichtsvoll. Der Versuch mit Rotkehlchen habe versagt.

Sodann erhält Herr Braun (Marienburg) das Wort zu einem Vortrage über den extranuptialen Gesang der Vögel (ausführlicheres Referat am Schlusse dieses Heftes).

Herr v. Lucanus hält den Gesang als Anlockungsmittel für das Weibchen doch für bedeutender, als der Redner annimmt, und erläutert dies an Beispielen. Redner unterscheidet automatische Gesänge und Gesänge die erst gelernt werden müssen (Schlag). Ein Buchfink z. B., jung aus dem Neste genommen, bleibt ein Stümper, während der Weidenlaubsänger und sogar der Baumpieper ihre Gesänge vollkommen reproduzierten, selbst wenn sie jung aus dem Nest genommen wurden. Der Redner ist der Ansicht, dafs der Gesang vom Sexualleben nicht zu trennen sei.

Auch Herr Kollibay vertritt die Ansicht, dafs der Gesang mit dem Sexualleben verbunden sei, wie ihn Beobachtungen am Girlitz belehrten. Es äufsern sich noch die Herren Stoll und v. Berlepsch zum gleichen Thema. Ersterer erwähnt, dafs er einen Hakengimpel und einen Buchfinken im Bauer gehabt habe und dafs der Buchfink den Gesang des Hakengimpels angenommen hatte. Letzterer meint, dafs das Singen von Zaunkönig und Wasseramsel im Winter die Ansicht, dafs der Gesang sexualen Ursprungs sei, durchkreuzten. Dem widerspricht Herr v. Lucanus und bemerkt noch, dafs die indische Schamadrossel für den Kampfruf eine besondere Melodie habe.

Hierauf spricht Herr Helm über den Zug des Stars. Der Vortragende widerspricht der Ansicht Gätkes, dafs die jungen Stare vor den alten fortziehen. Er weist an einer grofsen Zahl von Beobachtungen in Sachsen und auf mehreren Nordseeinseln nach, dafs Junge und Alte gemischt bis Ende September und Anfang Oktober vereint sich hier aufhalten. (Siehe weiter unten).

Nachdem Herr Kollibay dem Vortragenden gedankt, macht Herr Schalow noch auf einige Bücher aufmerksam, die er im Museumssaal ausgelegt hat, wo sie Interessenten besichtigen könnten. Ferner macht Herr Schalow auf Tierbilder aufmerksam, die nach 
der Mittagspause den auswärtigen Mitgliedern stereoskopisch vorgeführt werden sollen. Die Bilder wären einzig in ihrer Art und sind von Herrn Heinroth dem Redner übergeben worden. Herr Heck bemerkt, dafs ein Amsterdamer Präparator die Aufnahmen gemacht habe, sie hätten den ersten Preis von der Verlagshandlung Voigtländer erhalten.

Nach der Mittagspause photographierte Herr Stoll die Teilnehmer der Jahresversammlung, welche auf der Museumstreppe sich gruppiert hatten (das Bild kann gegen Einsendung von 70 Pf. in Briefmarken vom Generalsekretär bezogen werden), sodann zeigt Herr Schalow einigen Teilnehmern die vorerwähnten Bilder. Um 3 Uhr beginnt Herr Hantzsch seinen Vortrag „Über das Studium der arktischen Vögel". Der Begriff der arktischen Vögel kann verschieden gefalst werden. Schalow behandelt in seiner Arbeit über „Die Vögel der Arktis" Jena 1904, alle diejenigen Arten, die im nördlichen Polarmeer und dessen Inselgebieten vorkommen; viele andere nehmen die Grenze des Baumwuchses für abschliefsend an usw. Der Redner hält sich nicht an eine bestimmte zoogeographische Grenzlinie, sondern versteht unter den arktischen Vögeln die Arten, deren Verbreitungszentrum gegenwärtig in arktischen Gebieten liegt. Die endgültige Feststellung der hierher gehörigen Spezies ist keineswegs einfach, bedarf vielmehr bei manchen noch sorgfältiger Untersuchung. Gewisse Gattungen und Arten, wie Lagopus, Acanthis (linaria), Hierofalco (gyrfalco) gehören zweifellos hierher und müssen in all ihren Formen in Betracht gezogen werden, auch wenn solche weit südlichere Gebiete (Hochgebirge) bewohnen. Bei anderen, wie Corvus corax, der zwar in sehr hohe Breiten vordringt, aber fast kosmopolitisch auftritt, ist es fraglich, wo deren Verbreitungszentrum $\mathrm{zu}$ suchen ist. Gewisse rein arktische Arten brüten verhältnismäfsig weit nach Süden hinab (z. B. Fratercula, Somateria), andere nicht rein arktische Vögel wieder gehen weit nach Norden hinauf, z. B. Saxicola oenanthe und Anas boschas. Es ist deshalb nach Ansicht des Redners nicht richtig, nach blofs äufserlichen Gesichtspunkten diejenigen Vogelarten als arktische zu bezeichnen, deren Verbreitungsgebiet bis in die geographisch nördliche kalte Zone hineinreicht, umgekehrt solche auszuschalten, die, wenn auch in etwas anderen Formen, weit nach Süden vordringen.

Aufgabe des Studiums der arktischen Vögel wird nun zunächst sein, zu untersuchen, ob und inwieweit sich die hochnordischen Vertreter von den südlicher wohnenden unterscheiden und im positiven Falle eine durch trinäre Benennung hervorgehobene Kennzeichnung eintreten zu lassen (Fratercula arctica arctica (L.) und F. a. glacialis Steph., Cepphus grylle grylle (L.) und C. g. mandtii (Licht.).) Selbst Arten, die ursprünglich kaum nordische gewesen sein dürften, wie Saxicola oenanthe, haben sich subspezifisch verändert, hier in $S$. o. leucorhoa (Gm.). Das Studium 
dieser Unterschiede ist noch keineswegs abgeschlossen, wie neuerdings wieder die Veröffentlichungen über die Abtrennung der grönländischen Stockente (Anas boschas conboschas Brehm $=A$. $b$. spilogaster Schiöler) von der mitteleuropäischen zeigt. Die isländischen Vertreter dieser Art scheinen übrigens, soweit Redner Bälge untersuchen konnte, mit der europäischen Form, die Labradorvögel, ja vielleicht die Amerikaner überhaupt, mit der grönländischen übereinzustimmen.

Beim Untersuchen und Vergleichen von arktischen mit paläarktischen oder nearktischen Vögeln sind von vorn herein gewisse Gesichtspunkte zu berücksichtigen. Ganz abgesehen davon, dafs man natürlich nur Brut-, nicht Duchzugvögel der verschiedenen Örtlichkeiten in derselben Entwicklung des Gefieders in Betracht ziehen wird, ist auf das Wohngebiet selbst Wert zu legen. Zeigt es sich, dafs in einigen Ländern des Nordens gewisse Arten als Standvögel im weiteren Sinne auch während des Winters verweilen, ist deren spezifische Verschiedenheit gegenüber anderen Gebieten höchst wahrscheinlich. Der Redner bespricht einzelne Gebiete in dieser Hinsicht. Die verschiedenartige Entwicklung atlantischer und pazifischer Formen ist natürlich aufserdem zu berücksichtigen.

Die nordischen Formen erweisen sich gegenüber südlicheren im allgemeinen als stärker und widerstandsfähiger (vergl. Fratercula, Corvus corax, Acanthis linaria, Anas boschas, Lagopus in seinen Standvögelformen etc., mitunter aber auch als schlanker, geschmeidiger und daher beweglicher (vergl. Branta canadensis canadensis und B. c. hutschinsii, Cepphus grylle grylle und C. $g$. mandtii).

Wohl zu beachten ist bei der Untersuchung von Grölsenverhältnissen die individuelle Schwankung, die sich besonders bei nordischen Seevögeln in hohem Grade findet, und ihre einfache Erklärung in klimatischen und Nahrungsverhältnissen hat (vergl. Fulmarus glacialis glacialis (L.) und F.g. minor Kjärb.).

Auch die stärkere Ausblassung des Sommergefieders in den schnee- und eisbedeckten arktischen Gebieten gegenüber milderen schneefreien Klimaten ist $\mathrm{zu}$ berücksichtigen. Sie zeigt sich bei manchen Vögeln, besonders Möwen, recht auffällig und kann als Analogie zu den hochnordischen Lagopus-Individuen angesehen werden, bei denen besonders die weniger versteckt lebenden Männchen das weifse Winterkleid überhaupt selten völlig verlieren.

Aufser derartigen Untersuchungen über etwaige subspezifische Verschiedenheiten arktischer und in südlicheren Breiten wohnender Vogelarten, deren sorgfältige Kenntnis auch für die Beurteilung des Vogelzuges bedeutsam wird, ist es von grofsem Interesse, die Biologie der nordischen Vögel besser kennen zu lernen als bisher, zumal eine Menge dieser Arten als Durchzügler und Wintergäste nicht nur einem kleinen Kreise von Spezialornithologen, sondern einem grofsen Teile der Bevölkerung überhaupt bekannt sind. Wie wenig wissen wir über das Sommerleben vieler 
Strandläuferarten, z. B. Tringa canutus und subarquata oder Calidris arenaria, die als gewöhnliche Gäste alljährlich unsere Meeresküsten aufsuchen. Das Studium ihres Brutgeschäftes, der Aufzucht der Jungen und dieser selbst, die Zugverhältnisse an den Sommerwohnplätzen, sowie vieles andere, bedarf bei einer grofsen Reihe von Arten gar sehr der Aufklärung, und der Naturforschung steht hier noch ein weites Feld offen.

Nur in wenigen günstig gelegenen arktischen Gebieten, die schon verhältnismäfsig zeitig im Jahre eisfrei und für die Schifffahrt zugänglich werden, z. B. Spitzbergen, oder an solchen Örtlichkeiten, wo grölsere Expeditionen längere Jahre Aufenthalt genommen haben, oder wo dauernd gebildetere Weifse wohnen, wie in Grönland, sind bisher sorgfältige und planmäfsige Untersuchungen in dieser Beziehung angestellt worden, aus anderen Gebieten liegen zum grofsen Teil nur zufällige und nebenbei gesammelte Beobachtungen und Objekte vor, die noch dazu in der Hauptsache aus derselben Jahreszeit, den wenigen Sommermonaten, stammen. Wie dürftig aber auch derartiges Material ist, zeigt die Schwierigkeit, arktische Vogelbälge und Eier im Handel zu erwerben, und ein guter Teil dieser letzteren stammt noch dazu aus der Gefangenschaft oder ist, weil von Nichtornithologen gesammelt, ungenügend identifiziert.

Alle diese, hier nur in Kürze angedeuteten Gründe veranlafsten den Vortragenden, dem Studium der arktischen Avifauna näher zu treten. Die Leichtigkeit desselben liegt in der verhältnismälsig geringen Zahl der in Frage kommenden Spezies, die Schwierigkeit in dem Mangel an reichlichem, guten Material, der Unsicherheit der teilweise sehr alten Litteratur und den Hindernissen, die eigenen Forschungen entgegenstehen, zumal solche nur dann planmälsig angestellt werden können, falls jahrelange Tätigkeit, nicht nur wenige Sommermonate, diesen Gebieten gewidmet werden. Für gegenwärtig besonders vielversprechende Örtlichkeit in dieser Hinsicht hält Redner das südlichere BaffinLand, doch machen sich vor einer Bereisung aufser sorgfältigen theoretischen Vorbereitungen auch vielerlei praktische Erfahrungen nötig, ohne die eine erfolgreiche Expedition zweifelhaft wäre. Der Vortragende besuchte deshalb im Jahre 1906 das nordöstlichste Labrador. Über die ornithologischen Ergebnisse dieser Vorbereitungsreise wird in einer besonderen Arbeit berichtet werden. Der Redner schildert deshalb diese Reise nur in Kürze, hofft aber, dafs aus ihr eine spätere grölsere Expedition zur Erforschung der arktischen Vögel hervorgehen wird.

Nachdem die Versammlung dem Redner ihren Beifall gezollt hatte, dankte Herr Kollibay demselben für den vortrefflichen Vortrag und wies darauf hin, dafs, wer Schalow's „Vögel der Arktis" gelesen habe, erkennen müsse, wieviel in jenem Gebiete noch zu tun sei. Deshalb sei es sehr anzuerkennen, dafs Herr

Journ. f. Orn. LVI. Jahrg. Januar 1908. 
Hantzsch sich die Erforschung eines so wenig bekannten Landes vorgenommen habe.

Auch Herr Schalow gab seiner Freude darüber Ausdruck, dafs Herr Hantzsch gerade diese Gegenden besuchen will und empfiehlt die nachdrücklichste Unterstützung zur Verwirklichung dieser Pläne, an deren erfolgreicher Durchführung bei der von Herrn Hantzsch bewiesenen Willensstärke und Ausdauer nicht zu zweifeln sei. Auch geographische Kreise müsse man gewinnen.

Hiermit schliefst Herr Kollibay die Diskussion. Die Herren Schalow, Reichenow und Kollibay ziehen ihre Vorträge wegen der vorgerückten Stunde zurück. Zur Vorlage kommt noch eine Abhandlung des Herrn Buturlin über die Verbreitung der Vögel in NordostSibirien (wird später abgedruckt). Herr Kollibay dankt unter Zustimmung der Versammlung dem bisherigen Vorstande, insbesondere Herrn Reichenow für die Vorbereitung der so aufserordentlich erfolgreich verlaufenen Jahresversammlung und schliefst die Sitzung.

K. Kothe.

O. Haase.

\section{Über den extranuptialen Gesang und das Phänomen des Spottens.}

Referat von Fritz Braun über seinen am 6. X. 1907 gehaltenen Vortrag.

So viele sich auch Mühe geben, die Aufgaben des Gesanges in dem Leben der Vögel zu finden, immer wieder kommen Leute, die, ohne sich irgendwie um das Ergebnis dieser Arbeit zu kümmern, schlankweg behaupten: "Wir wollen von dem Streben jener, die die Natur nur mit dem Verstande auffassen und allen Naturerscheinungen grübelnd entgegentreten, nichts wissen. Jene Forscher versündigen sich an der Schönheit der Natur, da war Göthe doch ein ganz andrer Mann. Hatte er nicht Recht, von seinem Sänger zu behaupten:

„Ich singe, wie der Vogel singt,

der in den Zweigen wohnet.

Das Lied, das aus der Kehle dringt

ist Lohn, der reichlich lohnet?"

Sie vergessen dabei ganz, dafs die Naturwissenschaft mit Begriffen und nicht mit Gefühlen arbeitet, dafs ihre Aufgabe darin besteht, diese Begriffe immer schärfer herauszuarbeiten und das Netz der kausalen Beziehungen, die sie verbinden, allmählich zu entwirren.

Unsere Kritiker handeln ähnlich wie ein Mann, der dem Botaniker sagen wollte: „Lafs ab, die Blumen danach zu ordnen, auf welche Weise sie befruchtet werden. Viel höher als diese Lebensbeziehungen steht mir der Blumen Schönheit." Wie jenem Manne, müssen wir auch unseren Richtern antworten: „Gehet zu den Aesthetikern, bei den Naturforschern ist nicht euer Platz. 


\section{$2 \mathrm{BHL}$ Biodiversity Heritage Library}

1908. "Bericht über die 57. Jahresversammlung der Deutschen

Ornithologischen Gesellschaft 1907." Journal

$f u$

r Ornithologie 56, 122-146. https://doi.org/10.1007/bf02089685.

View This Item Online: $\underline{\text { https://www.biodiversitylibrary.org/item/43348 }}$

DOI: https://doi.org/10.1007/bf02089685

Permalink: https://www.biodiversitylibrary.org/partpdf/142667

\section{Holding Institution}

MBLWHOI Library

\section{Sponsored by}

MBLWHOI Library

\section{Copyright \& Reuse}

Copyright Status: No known copyright restrictions as determined by scanning institution.

This document was created from content at the Biodiversity Heritage Library, the world's largest open access digital library for biodiversity literature and archives. Visit BHL at https://www.biodiversitylibrary.org. 